\title{
Multi-sensor Data Real-time Monitoring and Management System Based on Onboard UAV for Ocean Observation
}

\author{
Xuan $\mathrm{Li}^{1, \mathrm{a}}$, Yong Han ${ }^{2, \mathrm{~b}}{ }^{*}$,Fang-Jie $\mathrm{Yu}^{3, \mathrm{c}}$, Ge Chen ${ }^{4, \mathrm{~d}}$ \\ ${ }^{1}$ College of Information Science \& Engineering, Ocean University of China, Qingdao 266100, \\ China; \\ ${ }^{2}$ Laboratory for Regional Oceanography and Numerical Modeling, Qingdao National Laboratory for \\ Marine Science and Technology, Qingdao 266100, China; \\ ${ }^{a}$ IIIxxx215@163.com, ${ }^{\text {b }}$ chinahanyong@126.com, ${ }^{c}$ fangjie.yu@gmail.com, ${ }^{d}$ gechen@public.qd.sd.cn
}

Keywords: UAV, ocean observation, monitoring and management system, multi-sensor, real-time

\begin{abstract}
It is of great significance for human beings to explore and develop marine resources. Nowadays, Unmanned Aerial Vehicle (UVA) is reaching a point where it can be used in marine environment research with multi-sensors. Abundant marine data acquisition, transmission, management in real-time are preconditions in the exploring and developing of marine resources with UAVs. In meet to these emerging demands, this paper presents a new data real-time monitoring and management system, multi-sensor data real-time monitoring and management system based on UAV for ocean observation, for marine environment monitoring. In this system, an UAV, equipped with four kinds of sensors, captures marine information in a flight mission, data are then transmitted toward the ground station for processing and displaying in real-time. In this paper, we discuss the system hardware architecture firstly, then a genetic architecture of system software is presented, details regarding several techniques used in dealing with sensor data are outlined. In addition, process of data playback in data management is analyzed. Finally, numerical flight experiments are implemented, and the results have shown feasibility of the software system.
\end{abstract}

\section{Introduction}

UAVs equipped with sensors has been applied more and more wider in civilian side, (e.g., in emergent situation, condition monitoring) for its advantages of light weight, flexibility and so on. Kyoungah and Impyeong developed a UAV based close-range rapid aerial monitoring system which produce DEM and orthoimages for emergency response. Its sensor system included camera, laser scanner, GPS and IMU [1]. Masahiko et al. studied a UAV-based sensor web monitoring system to acquire detailed information suited for disaster and environment monitoring in which sensors including camera, gyroscope compass, laser range meter, GPS are mounted on a UAV [2]. In Chinese, there either have been many applications. Peng et al. developed a system for safety inspection and diagnosis in which a UAV carrying aerial camera, visible-light sensors and laser sensors, collected images of power lines [3]. Zhang et al. developed a system for wheat breeding information acquirement in which different sensors such as camera, multi-spectral camera, infrared thermal imager are loaded on the UAV flying over the farmland [4]. Some of these systems can acquire and transmit data in real-time, but the procedure of processing data is taken after tasks, and some can process and display the results of only one sensor not all the whole scene data.

In our study, an UAV is equipped with four sensors, namely, SAR (synthetic aperture radar), LiDAR (Light Detection And Ranging), aerial camera, temperature and humidity sensors and the other payload, collecting information around the marine area, transmitting them to the ground station in real-time, and our software received, processed these data synchronously instantly. At present, data acquisition is an essential part of the process of signal detection, data transmission is an indispensable integral part in the communication of airborne systems and ground stations [5]. Ocean sensor data real-time transmission system based on wireless network provide a new set of data acquisition and transmission system [6]. Once established, system is expected to contribute to explore and develop marine resources, monitor marine environment, detect marine target, predict 
disaster and so on. Fully utilizing the characteristic of multiple threads running simultaneously to implement multiple tasks [7], system achieves the tasks such as plenty of data reading, data review.

\section{System Hardware Architecture}

The hardware architecture(see Fig. 1) of the proposed multi-sensor data real-time monitoring and management system based on UAV for ocean observation consists of three parts, collector module, transmission module, receive module.

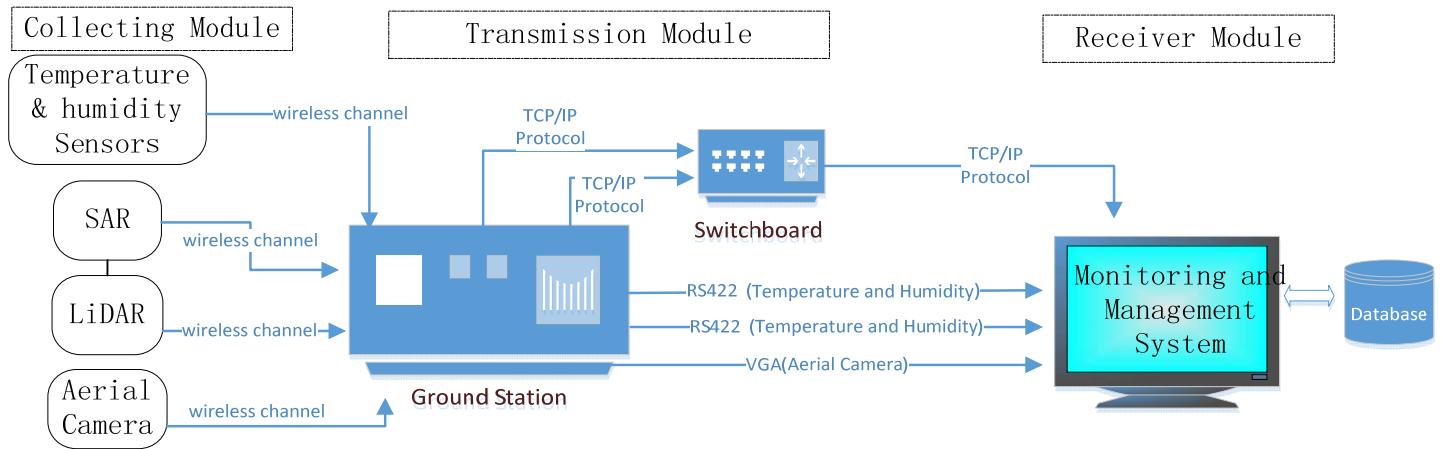

Fig.1 General view of the hardware architecture

Collector Module. To get ample information about the ocean in a fight mission, the collector module combines an aerial camera, temperature and humidity sensors, SAR and LiDAR, all of them are installed in the front of the UAV.

Transmit Module. Data transmission from UAV to base station through wireless channel, data signals are sent from UAV, a wireless signal receiver with an antenna receiving signal is put on the top of the base station. The temperature and humidity data are transferred by RS422 with ports, each temperature and humidity sensor is addressed using a unique port number. SAR and LiDAR data are transmitted via cables under TCP/IP protocol, and video transfer is based on VGA which receives analog signals.

Receive Module. The receive level is the software system, it is installed in a computer with high quality equipment. Different kinds of raw data are transmitted to here to be processed and displayed. The display area consists of four equirotal parts, respectively responsible for SAR picture display, LiDAR picture display, video player, curves of temperature and humidity. Management and control of the four kinds of sensor data in here simultaneously are the characteristics of the system. More comprehensive information though combining four kinds of data synchronously can be acquired.

\section{System Software}

Composition of System. The system is divided into two main modules(see Fig.2), Sensors Monitoring and Data Management. Part A, the Sensors Monitoring consists of two sub modules, being in charging of analyzing, processing and storage. Sub module D Settings, is a configuration part, supporting where the data should be stored. Part C, Data Monitor, can receive, process and display the data coming from the four sensors simultaneously, in the mean time all the data are stored in various tables designed specially in database with SQLServer (database management system ). Part B is divided into two parts, part $\mathrm{E}$ is regard to data searching through name and time. Data browsing and playing are in Part F. 


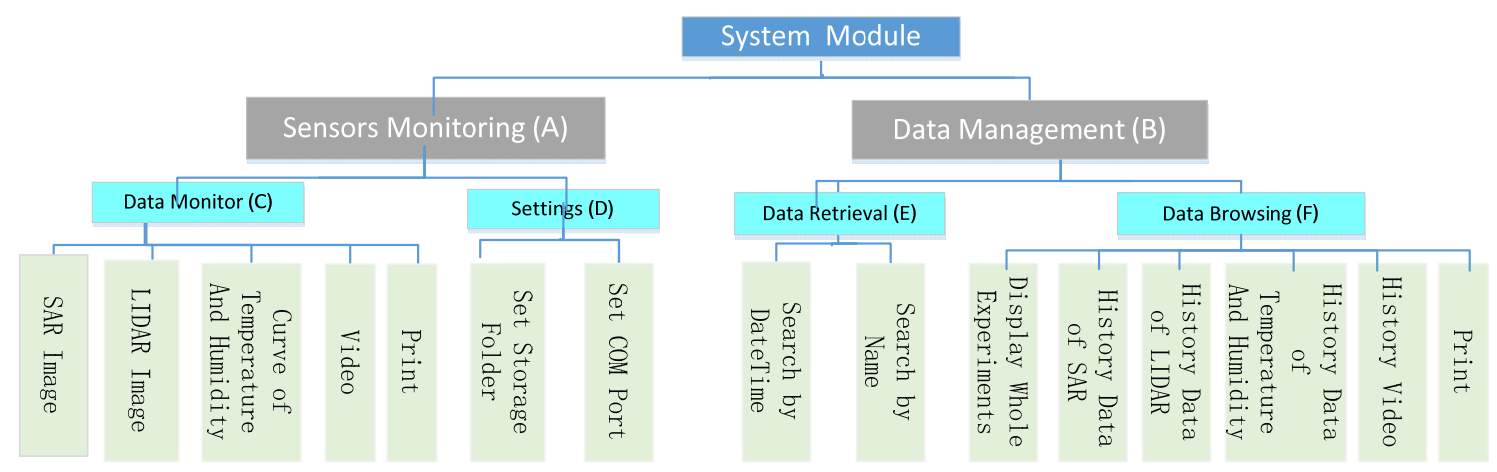

Fig.2 The functional module of system

Several Techniques Used in Data Processing. One of the most difficult and complicated segments is data integration in Date Monitor module, as well as in Data Browsing module, which consumes plenty of computation, the following techniques are proposed to arrange the task scheduling between each sub module.

Multi-thread and Asynchronous Operation Programming Technique. Each sensor sends data to the receiving system in inhomogeneous frequency, temperature and humidity detectors send data to receiving system every $0.7 \mathrm{~s}$, SAR and LiDAR data are generated in slower rate compared with temperature and humidity data, every 2 or 3 minutes, a new SAR image data or LiDAR zip file is produced. Aerial camera transmits video signal during the whole flight progress. Each data processing is a sub thread. In order to handle four kinds of data simultaneously and avoid interference, the multi-thread and asynchronous operation techniques are adopted. The multi-thread which carries out some duty in sub thread improving the hard real-time property of the system, boosting up the overall performance of the software system. The process of LiDAR data monitoring is as follows in Fig. 3.

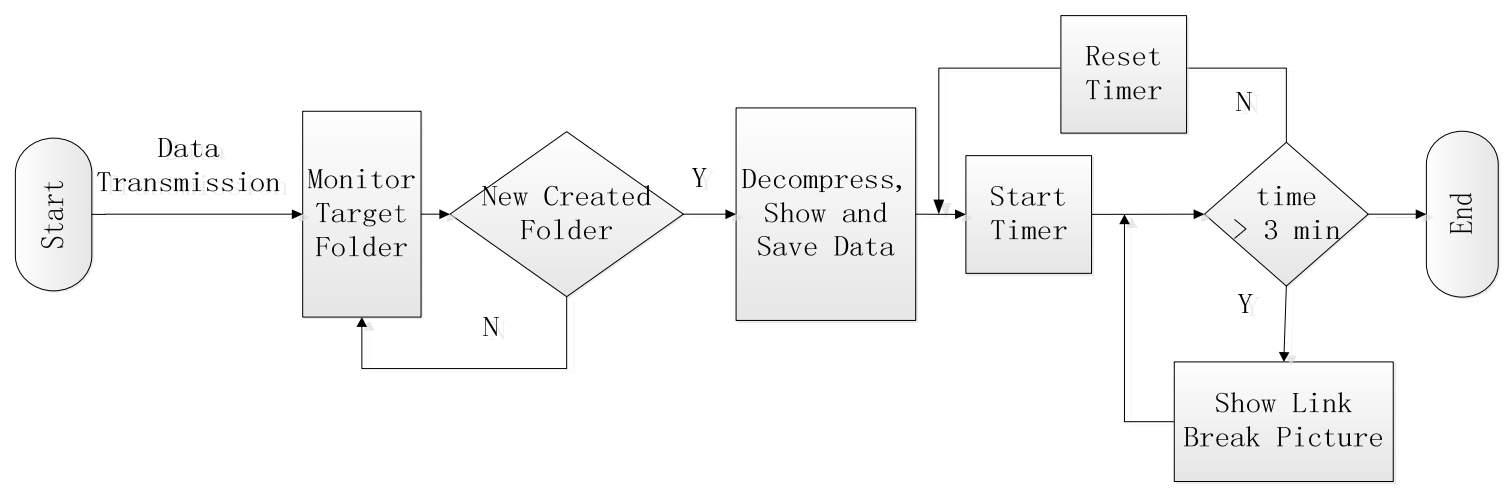

Fig. 3 General flow of monitoring files

The sub thread is provoked as the new data folders are created in the target folder, data of LiDAR are ZIP package which are decompressed automatically through programming control once detected by the FileSystemWatcher, the pictures data are displayed with PictureBox Control and stored into local folders in monitoring module. A Timer Control, starts timing before the next new data comes, if the time interval exceeds 3 minutes, a link disconnection picture meaning transmission broken is shown on the screen; then the Timer Control is reset for the next timing. In an analogous way, data of SAR are processed through the other sub threads.

AForge.NET Framework Implementation in Video Capture. As for the video, we opt AForge.NET, which is applied to domains such as computer vision, machine learning, image processing, etc.When a video capture card is connected to the computer as the system starts working, system can detect it with AForge.NET, then a sub thread starts to create a video file consisting of the video frame rate, resolution, etc. And the videoSourcePlayer Control captures the video and shows it. If there is no video capture card, or signal link breaks, a link break warning will be shown and a sub thread will be created to reconnect the video signal. This is illustrated in Fig. 4. 


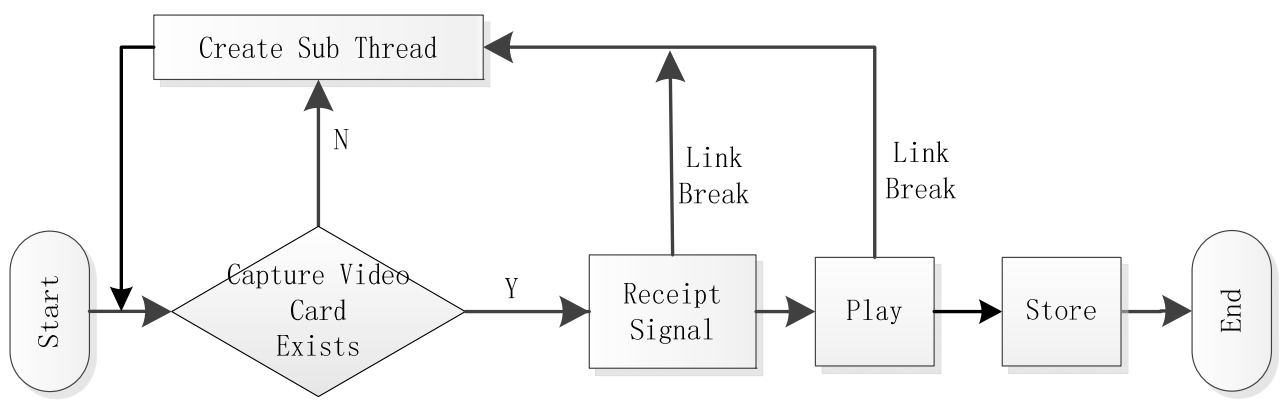

Fig. 4 Process of video capture

The Dynamic Douglas - Peucker Algorithm in Drawing Curves. The Chart Control executes once curve drawing when there comes a set of temperature data. In one mission there is a large amount of data owning to the high transmission rate $(0.7 \mathrm{~s})$, and some redundant data because of several reasons. Such as data collected when the UAV takes off, hovers in the air, these data change little, resulting in redundancy. So that the heavy workloads from the rising data number affects efficiency of drawing badly. To solve this problem, we remove the redundant data using an improved dynamic Douglas - Peucker algorithm.

We use a list to receive the new coming temperature and humidity data. The size of the list increases dynamically as the data are transmitted into it. Points whose increment or decrement of temperature is less than $0.01{ }^{\circ} \mathrm{C}$ compared with the previous point are removed. When the number of data is more than two, the third new data can trigger the dynamic Douglas - Peucker algorithm. We suppose the first two points P1, P2, which are selected to form a straight line, named Lp, let the Eq. (1) be the equation of Lp. We suppose the third point to be P3.

$\mathrm{Ax}+\mathrm{By}+\mathrm{C}=0$

Then we can obtain the perpendicular distance from P2 to Lp by the following Eq. (2): $\mathrm{d}_{2}=\frac{\left|A X_{2}+B Y_{2}+C\right|}{\sqrt{A^{2}+B^{2}}}$

You can see a simplified illustration in Fig. 5 below.

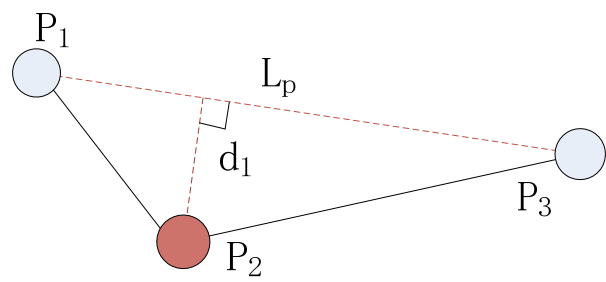

Fig. 5 Determination of key point

As the new points are put into the list continuously, the dynamic Douglas - Peucker algorithm is triggered repeatedly, every point has a corresponding calculated result, $d_{k}$ through Eq. (3), Point ${ }_{k}$ is the target point, Point $\mathrm{k}_{\mathrm{-}-1}$ and Point $\mathrm{k}_{\mathrm{k}+1}$ are the points before and after Point $\mathrm{k}_{\mathrm{k}}, \mathrm{d}_{\mathrm{k}}$ is the calculated result, Fig. 6 and Fig. 7 show us an illustration of more points. When the number of list is more than 1000, we begin to remove the less influencing point whose $d_{k}$ is minimum, keeping the list stay in a moderate capacity. After the whole calculation, all the key points are retained, the points that change little, have slight influence on the shape of the curve, are removed. The flow of Chart drawing in this stage is shown in Fig. 8.

Double area $=$ Math.Abs $\left(.5 *\left(\right.\right.$ Point $_{\mathrm{k}-1} . \mathrm{X} *$ Point $_{\mathrm{k}+1} . \mathrm{Y}+$ Point $_{\mathrm{k}+1} . \mathrm{X} *$

Point $_{\mathrm{k}} . \mathrm{Y}+$ Point $_{\mathrm{k}} . \mathrm{X} *$ Point $_{\mathrm{k}-1} . \mathrm{Y}-$ Point $_{\mathrm{k}+1} . \mathrm{X}^{*}$ Point $_{\mathrm{k}-1} . \mathrm{Y}-$ Point $_{\mathrm{k}} . \mathrm{X}^{*}$

Point $_{\mathrm{k}+1} . \mathrm{Y}-$ Point $_{\mathrm{k}-1} . \mathrm{X} *$ Point $\left.\left._{\mathrm{k}} . \mathrm{Y}\right)\right)$;

Double bottom $=$ Math.Sqrt $\left(\right.$ Math.Pow $\left(\right.$ Point $_{\mathrm{k}-1} \cdot \mathrm{X}-$ Point $\left._{\mathrm{k}+1} . \mathrm{X}, 2\right)+$

Math.Pow(Point ${ }_{\mathrm{k}-1} . \mathrm{Y}-$ Point $_{\mathrm{k}+1}$.Y, 2));

Double $\mathrm{d}_{\mathrm{k}}=$ area / bottom *2; 


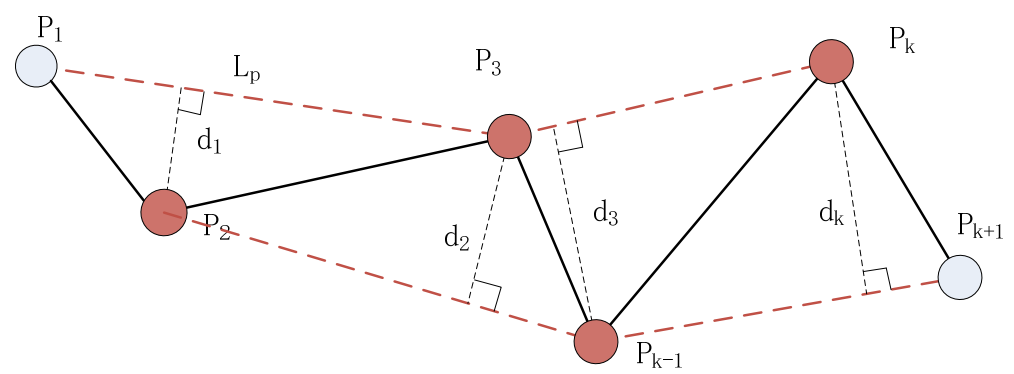

Fig. 6 Progress of calculation

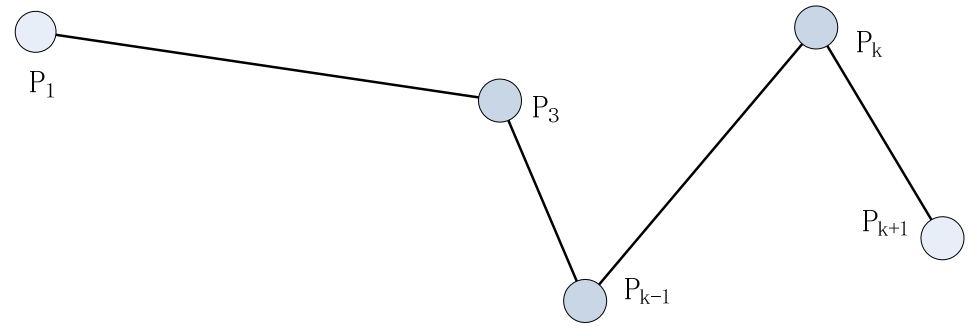

Fig. 7 Points after calculation and remove

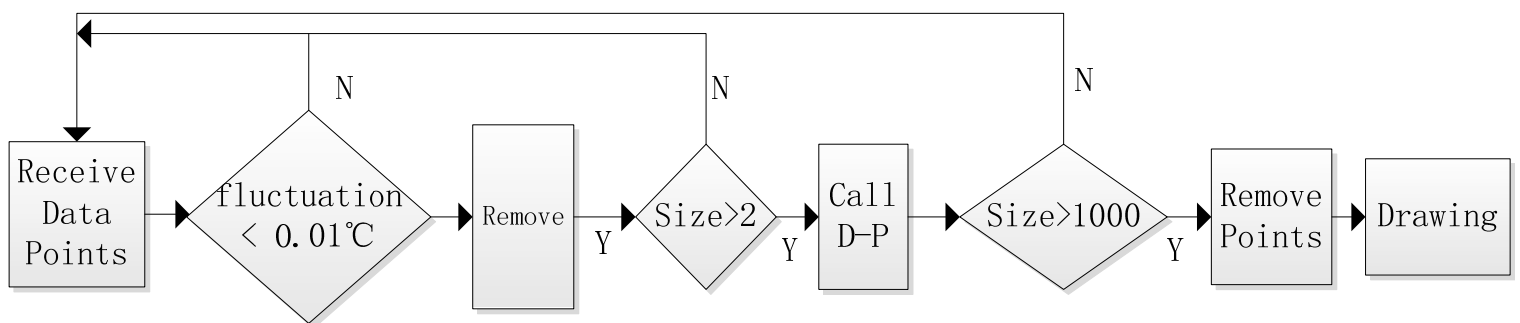

Fig. 8 Process of remove redundancy

With the aforementioned techniques, our software provides us an efficient, stable, flexible system in processing, monitoring and managing marine data.

\section{Flight Experiment}

We have taken flight experiments in The 60 office of China PLA General Political Department, Nanjing Province. During the UAV's flight, data are sent from UAV to the ground station, and then transmitted to the system in real-time. Testing results are shown in Fig. 9. We can get how the temperature and humidity changes through the curves and the conditions of wind fields by LiDAR and SAR images of one ocean area. Several useful flights are made to aid the calibration and optimization of system design, and we think the system will have a very good application in real flight mission. 


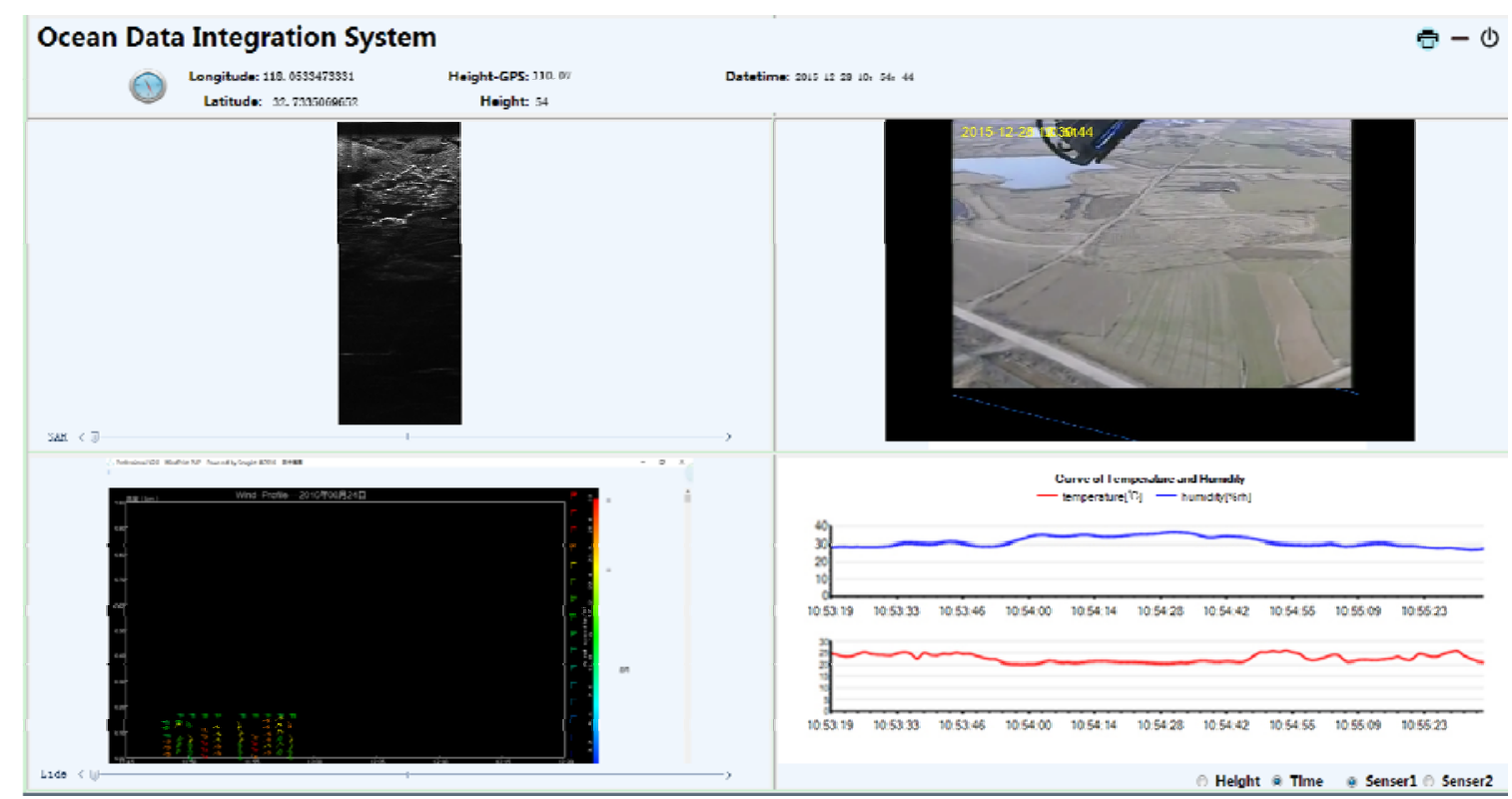

Fig. 9 The real-time monitoring platform

\section{Conclusion}

As we know, China is at the initial stage in airborne remote sensing in marine monitoring, so it is obvious that the application of UAV with airborne sensors in marine observation will emerge with a fast speeding, and it will be full of potential in exploring marine resources. How to receive, process and manage all the different valuable data from different sensors gathered through the UAV is crucial to researchers. In view of this, a new system is developed and implemented to collect and manage the environment data around marine area. This paper presents the hardware basis firstly, then introduces the composition of this system software. Several techniques used in data monitor and management modules are analyzed, and the procedure of data playback in data management is illuminated. The features of system are also presented. Finally, the results of flight experiments validate the applicability of this system in ocean observation.

\section{Acknowledgments}

This research is funded by The National High Technology Research and Development Program of China under Grant 2014AA09A511.

Programs: The National High Technology Research and Development Program of China (Grant NO. 2014AA09A511).

\section{Reference}

[1] Choi, K., \& Lee, I., A uav based close-range rapid aerial monitoring system for emergency responses. ISPRS - International Archives of the Photogrammetry, J. Remote Sensing and Spatial Information Sciences, XXXVIII-1/C22(1), (2012) 247-252.

[2] Nagai, M., Witayangkurn, A., Honda, K., and Shibasaki, R., Uav-based sensor web monitoring system. International Journal of Navigation \& Observation, (2012).

[3] Peng, X. Y., Chen, C., Rao, Z. Q., Yang, B. S., and Mai, X. M., Safety inspection and intelligent diagnosis of transmission line based on unmanned helicopter of multi sensor data acquisition. High Voltage Engineering, 41 (1) (2015) 159-166.

[4] Zhang, B., Luo, X., Lan, Y., Huang, Z., Zeng, M., and Li, J., Agricultural environment monitor system based on UAV and wireless sensor networks. Transactions of the Chinese Society of Agricultural Engineering, 31 (17) (2015) 176-182. 
[5] Luo, Y., Chen, X., Data acquisition and communication system of miniature UAV based on DSP. International Conference on Control Engineering and Communication Technology, (2012) 736 740 .

[6] Qu, L. C., Li, X., and Sun, F. J., System design for ocean sensor data transmission based on wireless networks. Netinfo Security, 2 (2015) 40-45.

[7] Zhou M. G., Yu Y. Y., and Zhou J. D., An electric power management information system based on multi-thread technology. Computer Era, 5 (2010) 35-36.

[8] Wang, D., Fang, G., Pan, D., Hao, Z., and Zhu, Q., Introduction to the airborne marine surveillance platform and its application to water quality monitoring in china. Acta Oceanologica Sinica, 29 (2) (2010) 33-39.

[9] Wang, S. M., Li, Q., and Hu, H. P., Establishment of offshore marine observation equipment sensor monitoring system. International Conference on Advances in Mechanical Engineering and Industrial Informatics (AMEII 2015), 15 (2015) 1380-1385.

[12]Peng, Y., Meng, F., and Chu, D., Electric power data acquisition system based on multi-thread mechanism. 2013 International Conference on Cyber-enabled Distributed Computing and Knowledge Discovery, IEEE Computer Society, 8215 (2013) 273-276.

[13]Tao, M. L., Chen, D. F., Lu, Q. G., Shu, L., and Zhao, Y. P., Real-time virtual instrument platform based on multi-thread technique. Advanced Materials Research, 468-471(2012) 2903-2907.

[14] Yang, G. J., Li, C. C., Yu, H. Y., Xu, B., Feng, H. K., Gao, L., and Zhu, D. M., UAV based multi-load remote sensing technologies for wheat breeding information acquirement. Transactions of the Chinese Society of Agricultural Engineering, 31 (21) (2015) 184-190.

[15]Zhou, F., Zheng, W., and Wang, Z., Real-time motion estimation for UAVs based on dissimilar multi-sensor data fusion. Jiqiren/robot, 37 (1) (2015) 94-101.

[16] Abidin, A. F. Z., Jusoh, M. H., James, E., Junid, S. A. M. A., and Yassin, A. I. M., Real-Time remote monitoring with data acquisition system. IOP Conference Series: Materials Science and Engineering, 2015.

[17]Dymarczyk, M., Design of a remote data monitoring system based on sensor network. International Journal of Smart Home, 9 (5) (2001) 216-218.

[18]Hu, S. Z., Design of a multi-thread data collection system. Applied Mechanics \& Materials, 249-250 (2012) 1259-1263.

[19]Dalla Mura, M., Benediktsson, J. A., Chanussot, J., Gamba, P., Pacifici, F., and Prasad, S., Challenges and opportunities of multimodality and data fusion in remote sensing. Proceedings of the IEEE, 103 (9) (2015) 1585-1601.

[20]Hummel, G., Russ, M., Stütz, P., Soldatos, J., Rossi, L., and Knape, T., et al. Intelligent multi sensor fusion system for advanced situation awareness in urban environments. Future Security, Springer Berlin Heidelberg, 318 (2012) 93-104.

[20]Li, M. O., Wang, X. M., and Wen-Bo, N. I., Design of data acquisition and display system based on DSP. Instrument Technique \& Sensor, (7) (2011) 41-43.

[21]Li, W. H., Li, Y., Yu, P., Gong, J. H. and Shen S., The Trace Model: A Model for simulation of the tracing process during evacuations in complex route environments. Simulation Modelling Practice \& Theory, 60 (2016) 108-121.

[22]Naeem, W., Xu, T., Sutton, R., and Tiano, A., The design of a navigation, guidance, and control system for an unmanned surface vehicle for environmental monitoring. Proceedings of the Institution of Mechanical Engineers Part $M$ Journal of Engineering for the Maritime Environment, 222 (2) (2008) 67-79. 
[23]Peng, X. J., Yang, K. T., Study on a novel marine data acquisition system, Design, Manufacture and Mechatronics (ICDMM 2015), (2015) 895-903.

[24]Al-Asadi, T. A., and Baiee, W. R., Improved Douglas-Peucker algorithm with dynamic threshold values. International Journal of Digital Content Technology \& Its Applic, 8 (6) (2014) 61-67.

[25]Visvalingam, M., Whyatt, J. D., The Douglas-Peucker algorithm for line simplification: re-evaluation through visualization. Computer Graphics Forum, Elsevier North-Holland, Inc..9 (3) (1990) 213-228.

[26]Xie, Z., Wang, H., and Wu, L., The improved Douglas-Peucker algorithm based on the contour character. IEEE, 1-5 (2011).

[27]Li, L., and Jiang, W., An improved Douglas-Peucker algorithm for fast curve approximation. Image and Signal Processing (CISP), 2010 3rd International Congress on IEEE, 4 (2010) 1797-1802. 